\title{
Invasive neuromonitoring with an extended definition of delayed cerebral ischemia is associated with improved outcome after poor-grade subarachnoid hemorrhage
}

\author{
Michael Veldeman, MD, ${ }^{1}$ Walid Albanna, MD, ${ }^{1}$ Miriam Weiss, MD, ${ }^{1}$ Catharina Conzen, MD, ${ }^{1}$ \\ Tobias Philip Schmidt, MD, ${ }^{1}$ Henna Schulze-Steinen, MD, ${ }^{2}$ Martin Wiesmann, MD, ${ }^{3}$ \\ Hans Clusmann, MD, ${ }^{1}$ and Gerrit Alexander Schubert, MD ${ }^{1}$
}

\begin{abstract}
Departments of ${ }^{1}$ Neurosurgery, ${ }^{2}$ Intensive Care Medicine, and ${ }^{3}$ Diagnostic and Interventional Neuroradiology, RWTH Aachen University, Aachen, Germany
\end{abstract}

\begin{abstract}
OBJECTIVE The current definition of delayed cerebral ischemia $(\mathrm{DCI})$ is based on clinical characteristics precluding its use in patients with poor-grade subarachnoid hemorrhage (SAH). Additional concepts to evaluate the unconscious patient are required. Invasive neuromonitoring (INM) may allow timely detection of metabolic and oxygenation crises before irreversible damage has occurred.

METHODS The authors present a cohort analysis of all consecutive SAH patients referred to a single tertiary care center between 2010 and 2018. The cohort $(n=190)$ was split into two groups: one before $(n=96)$ and one after $(n=94)$ the introduction of INM in 2014. A total of 55 poor-grade SAH patients were prospectively monitored using parenchymal oxygen saturation measurement and cerebral microdialysis. The primary outcome was the Glasgow Outcome Scale-Extended (GOSE) score after 12 months.

RESULTS With neuromonitoring, the first $\mathrm{DCl}$ event was detected earlier (mean 2.2 days, $p=0.002$ ). The overall rate of DCl-related infarctions decreased significantly (from $44.8 \%$ to $22.3 \% ; p=0.001$ ) after the introduction of invasive monitoring. After 12 months, a higher rate of favorable outcome was observed in the post-INM group, compared to the pre-INM group (53.8\% vs 39.8\%), with a significant difference in the GOSE score distribution (OR 4.86, 95\% $\mathrm{Cl}-1.17$ to $-0.07, p=0.028)$.

CONCLUSIONS In this cohort analysis of poor-grade SAH patients, the introduction of INM and the extension of the classic $\mathrm{DCl}$ definition toward a functional dimension resulted in an earlier detection and treatment of $\mathrm{DCl}$ events. This led to an overall decrease in $\mathrm{DCl}$-related infarctions and an improvement in outcome.
\end{abstract}

https://thejns.org/doi/abs/10.3171/2020.3.JNS20375

KEYWORDS subarachnoid hemorrhage; invasive neuromonitoring; brain tissue oxygen saturation; cerebral microdialysis; delayed cerebral ischemia; vascular disorders

$\mathrm{A}$ NEURYSMAL subarachnoid hemorrhage ( $\mathrm{SAH})$ is a devastating disease with persistent high morbidity and mortality rates. Due to its high incidence in young patients, SAH carries a socioeconomic burden comparable to that of ischemic stroke. ${ }^{1}$ Patients surviving the initial hemorrhage remain susceptible to further ischemic damage for up to 3 weeks. Ischemic complications occurring in this phase have been coined as delayed cerebral ischemia (DCI). The occurrence of DCI and DCI-related infarctions is the main contributor to SAH mortality and morbidity. ${ }^{2}$ Angiographic large-vessel cerebral vasospasm was historically considered to be the sole cause of DCI. ${ }^{3}$ Vasospasm, however, occurs in about two-thirds of SAH patients, of whom only half will develop actual DCI. The remaining half will remain asymptomatic without the development of infarctions despite considerable vessel narrowing. These observations are congruous with results from large randomized trials such as the CONSCIOUS trials in which effective reversal of vasospasm did not result in superior outcome. Conversely, nimodipine im-

ABBREVIATIONS CLINA = continuous local intraarterial nimodipine application; $\mathrm{CMD}=$ cerebral microdialysis; $\mathrm{DCl}=$ delayed cerebral ischemia; $\mathrm{GOSE}=\mathrm{Glasgow}$ Outcome Scale-Extended; $\mathrm{H} \& \mathrm{H}=$ Hunt and Hess; $\mathrm{iHTN}=$ induced euvolemic arterial hypertension; $\mathrm{INM}=$ invasive neuromonitoring; $\mathrm{p}_{\mathrm{ti}} \mathrm{O}_{2}=$ brain tissue oxygenation; $\mathrm{SAH}=$ subarachnoid hemorrhage.

SUBMITTED February 6, 2020. ACCEPTED March 16, 2020.

INCLUDE WHEN CITING Published online May 15, 2020; DOI: 10.3171/2020.3.JNS20375. 
proves clinical outcome with only a limited effect on angiographic vasospasm. ${ }^{4,5}$ Furthermore, cerebral infarction correlates with the territory of angiographic vasospasm in only $25 \%-81 \%$ of SAH cases ${ }^{6,7}$ Additional vascular or tissue-specific mechanisms need to be involved either by reduced oxygen/energy deliverance or increasing demand. Possible explanations for reduced ischemic tolerance have been investigated in animal research and include failure of cerebral autoregulation, cortical spreading depolarization, microvascular spasms, and formation of microthrombi. ${ }^{8-11}$

DCI has been defined as the occurrence of focal neurological impairment or a decrease in consciousness. ${ }^{12}$ This definition is based on clinical characteristics and therefore only applicable to neurologically evaluable patients. This largely precludes its use in poor-grade $\mathrm{SAH}$ patients who are at the highest risk for ischemic complications. ${ }^{13} \mathrm{Se}-$ quential imaging such as CT perfusion imaging allows for a snapshot of brain perfusion, aiding in the diagnosis of DCI. Despite recent advancements, radiation exposure remains high. ${ }^{14}$ Also, the need for transportation of critically ill patients and the risk of delayed diagnosis of impending ischemia underline the need for other monitoring techniques.

Invasive neuromonitoring (INM) allows for live, bedside assessment of brain oxygenation and metabolism. Brain tissue oxygenation $\left(\mathrm{p}_{\mathrm{ti}} \mathrm{O}_{2}\right)$ monitoring is strongly recommended for patients at risk of cerebral ischemia or hypoxia. Cerebral microdialysis (CMD) is recommended as an add-on to other monitoring modalities in SAH patients..$^{15}$

In 2014, INM consisting of $\mathrm{p}_{\mathrm{ti}} \mathrm{O}_{2}$ and CMD was introduced into our institutional treatment algorithm. In the context of endovascular rescue strategies, we were able to show that INM is a valuable tool to measure treatment efficacy. ${ }^{16,17}$ The purpose of this observational trial was to assess the impact of INM on the detection rate of DCI and its influence on patient outcome.

\section{Methods}

\section{Patient Population and Study Design}

This trial was designed as a cohort analysis from 2010 to 2018 in which we compare patients before (pre-INM) and after (post-INM) introduction of INM in 2014. All patients admitted to our institution with confirmed aneurysmal SAH in the respective time frame were screened for eligibility. We included all poor-grade SAH patients (Hunt and Hess [H\&H] grades 3-5) who were between 18 and 90 years of age. The $\mathrm{H} \& \mathrm{H}$ scale was preferred over the World Federation of Neurosurgical Societies scale due to its easiness in use, as no steps are required to establish the grade. To correct for postictal reduced consciousness, grading was allocated as the best clinical grade within 24 hours after admission. Patients were excluded in cases of relevant angiographic vasospasm upon admission, admittance more than 5 days after the ictus, or early death $(<7$ days postictus). Also excluded were patients with mycotic or trauma-induced pseudoaneurysms and aneurysms associated with arteriovenous malformations.

This observational trial was retrospectively registered at the ISRCTN registry (reference no. 16523175) on July
25, 2019 (https://doi.org/10.1186/ISRCTN16523175). The prospective data collection was registered on May 20, 2014, at ClinicalTrials.gov (NCT02142166).

The trial was conducted in accordance with the recommendations of the ethics committee of the medical faculty of RWTH Aachen University. Informed consent was obtained from all prospectively included patients.

\section{Standard Treatment Algorithm}

Our SAH treatment protocol has been published previously $^{16,17}$ (Fig. 1). In brief, after establishing the diagnosis of the hemorrhage, aneurysms were secured within 48 hours via surgical clipping or endovascular coiling. All patients were monitored in our neurointensive care unit. Failing an initial wake-up test, poor-grade patients underwent INM probe placement. Contraindications were coagulation disorders and predictable early death (brainstem damage on imaging or bilateral fixed pupils). Brain tissue oxygen probes (NEUROVENT PTO, RAUMEDIC) and CMD catheters (71 High Cut-Off Brain Microdialysis Catheter, $\mu$ dialysis) were placed unilaterally on the side of the ruptured aneurysm or, in cases of midline aneurysms, on the side with higher subarachnoid blood load. To yield information from two vascular territories (middle and anterior cerebral arteries), probes were placed in the frontal watershed region, $4.5 \mathrm{~cm}$ lateral to the midline in front of the coronal suture.

The occurrence of DCI was determined based on two diagnostic entities: classic or functional DCI.

\section{Classic DCl}

The criteria set by Vergouwen et al. were used to define classic DCI as a new focal neurological deficit or a decrease in Glasgow Coma Scale score $\geq 2$ for at least 1 hour, not ascribable to alternative diagnoses. ${ }^{12}$

\section{Functional $\mathrm{DCl}$}

Perfusion CT scanning was used in the pre-INM group for functional DCI assessment in unconscious patients. In the post-INM group, its use was confined to the evaluation of refractory DCI and DCI treatment surveillance. DCI was defined as a perfusion deficit with typical territorial cerebral blood flow/mean transit time mismatch.

Upon implementation of INM, the definition of DCI was extended to include oxygenation crises $\left(\mathrm{p}_{\mathrm{ti}} \mathrm{O}_{2}<10 \mathrm{~mm}\right.$ $\mathrm{Hg}$ ) or metabolic derangements (lactate/pyruvate ratio $\geq$ 40). After diagnosis, first-tier treatment was started, consisting of induced euvolemic arterial hypertension (iHTN; $\geq 180 \mathrm{~mm} \mathrm{Hg}$ ) by means of intravenous noradrenaline.

Cases refractory to iHTN were reevaluated by perfusion CT imaging. With persistent perfusion mismatch, second-tier endovascular rescue therapy was considered after interdisciplinary consensus. Transluminal balloon angioplasty was used in cases of proximal vasoconstriction and intraarterial spasmolysis in cases of diffuse vasospasm. Continuous local intraarterial nimodipine application (CLINA) was reserved as a third-/last-tier rescue therapy. Microcatheters (Excelsior SL-10, Stryker Neurovascular) were advanced into the internal carotid artery or vertebral artery. CLINA-treated patients received weight-adapted 


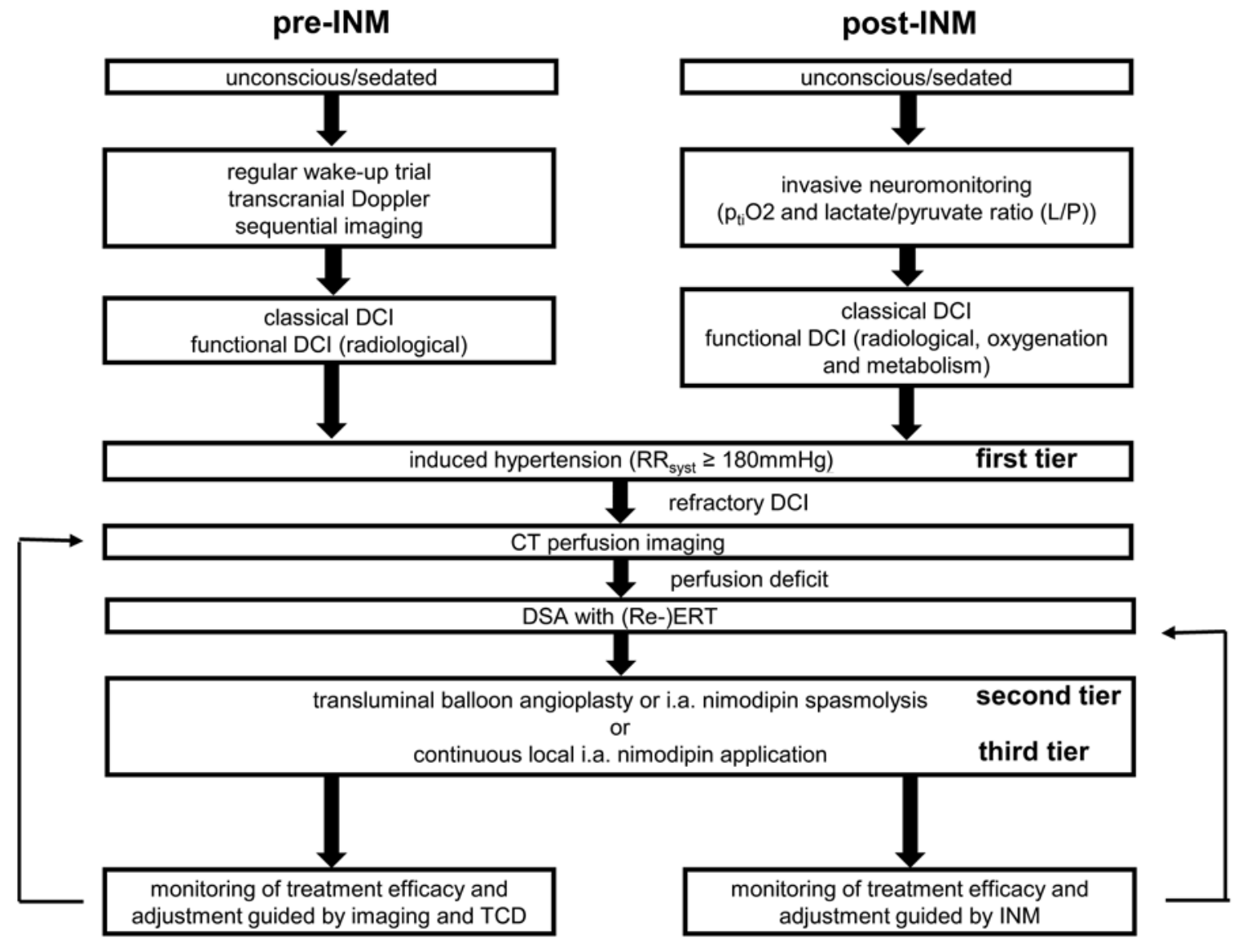

FIG. 1. Differences in the diagnostic algorithm but identical treatment algorithm between the pre-INM and post-INM groups. In the post-INM group, the additional functional dimension of $\mathrm{DCl}$ (oxygenation crises or metabolic derangement) was introduced. Functional DCl, in addition to a perfusion deficit (radiological), was defined by an oxygenation crisis $\left(\mathrm{p}_{\mathrm{ti}} \mathrm{O}_{2}<10 \mathrm{~mm} \mathrm{Hg}\right)$ or metabolic derangement (lactate/pyruvate ratio $\geq 40$ ) as measured by the INM probes. DSA = digital subtraction angiography; ERT = endovascular rescue therapy; i.a. = intraarterial; $\mathrm{RR}_{\text {syst }}=$ systolic blood pressure; $\mathrm{TCD}=$ transcranial Doppler.

intravenous tirofiban (Aggrastat, Correvio Pharma) and remained sedated until catheter removal. Independent of the ongoing treatment tier, therapy was reevaluated daily and de-escalated as soon as possible. The de-escalation process was guided by imaging in the pre-INM group and by imaging and INM in the post-INM group.

\section{Outcome}

The primary outcome measure was the Glasgow Outcome Scale-Extended (GOSE) score at 12 months after ictus. ${ }^{18}$ The GOSE score was dichotomized into unfavorable outcome (GOSE scores 1-4) and favorable outcome (GOSE scores 5-8). In the pre-INM group, outcome data were collected prospectively during regular follow-up visits. Missing information was appended either by analysis of patient files or by a structured telephone interview conducted by a blinded assessor. ${ }^{19}$ In the pre-INM group, the GOSE score at 12 months had to be reconstructed retrospectively by investigation of patient files or by contacting the patient, his or her next of kin, or caregiver by telephone in a structured interview.

The secondary outcomes were the following: GOSE score at discharge and at 6 months, death, lag between ic- tus and first treatment-triggering DCI event (days), number of CT investigations performed during the DCI time frame, prevalence of silent infarctions (i.e., the occurrence of a demarcated infarction as the first sign of ongoing DCI), rate of overall DCI-related infarctions, and DCIrelated mortality.

\section{Statistical Analysis}

In the dichotomous outcome analysis, the GOSE data were compared between groups using chi-square testing. Nondichotomized data were tested with an ordinal logistic regression model. The GOSE score was collapsed to a 6-point ordinal scale in which death and vegetative state, as well as lower and upper good recovery, were combined. In both analyses, the modified Fisher scale, H\&H grade, and DCI occurrence were added as predictor covariates. Sliding dichotomy was not considered due to its additional stratification based on expected prognosis, increasing the risk of information bias..$^{20}$ All data are presented as means and standard deviations for normally distributed continuous variables and as medians and interquartile ranges for nonnormally distributed continuous variables. Categorical variables are depicted as frequencies and proportions. 


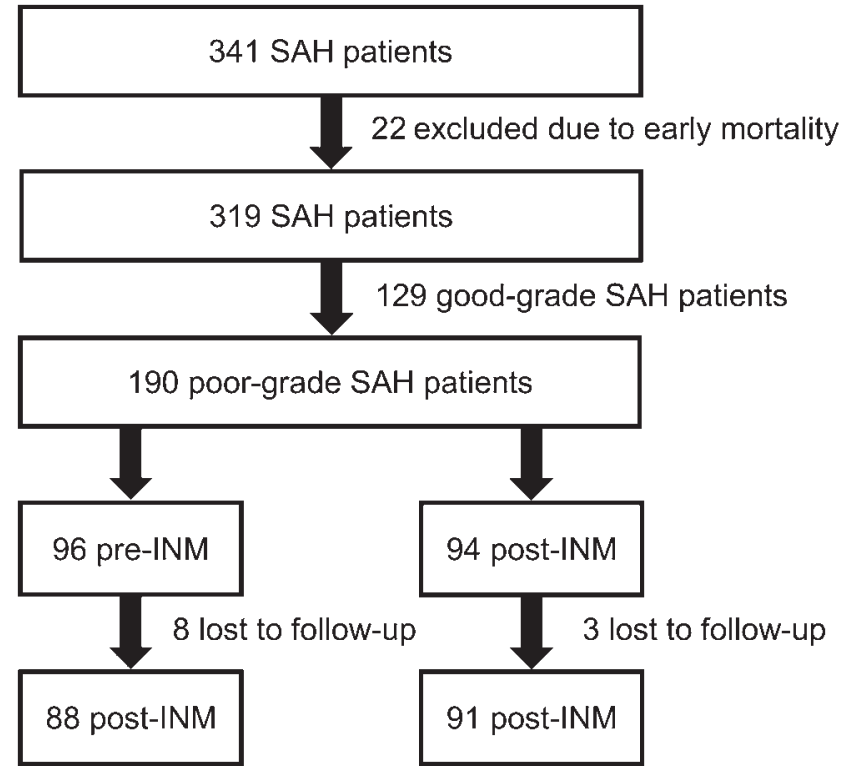

FIG. 2. Flowchart of patient recruitment.

After normality testing via plotting and the Shapiro-Wilk test, the appropriate statistical test was selected. For nominal data, the chi-square test was used; for normally distributed continuous data, the unpaired t-test was used; and for nonnormally distributed data, the Mann-Whitney Utest was used. Missing outcome data were not imputed. All statistical analyses were performed using IBM SPSS Statistics version 25 (IBM Corp.). Statistical significance was defined as a two-sided $\mathrm{p}<0.05$.

\section{Results \\ Patients}

A total of 341 consecutive patients with aneurysmal SAH were admitted to our department between January 2010 and December 2018. Due to early, not DCI-related, deaths (death in $<7$ days), 22 patients were excluded. Of the remaining 319 patients, 190 were clinically classified as having an SAH that was H\&H grade 3 or higher. Ninety-six patients meeting the inclusion criteria were admitted before (pre-INM) and 94 patients after (post-INM) invasive monitoring became available (Fig. 2). Baseline characteristics of the two study groups, summarized in Table 1 , were comparable, with a nonsignificant larger proportion of modified Fisher grade 3 hemorrhages in the postINM group of 58 patients $(61.7 \%)$ versus 44 patients in the pre-INM group $(45.8 \% ; \mathrm{p}=0.068)$. Additional variables were analyzed and are available in Supplementary Table 1. In the critical phase (days 7-14), 45 patients (46.9\%) in the pre-INM group were neurologically assessable, compared to 47 patients $(50.0 \%)$ in the post-INM group. INM was implemented in 55 patients $(58.8 \%)$. Forty-four patients $(46.8 \%)$ received dual monitoring $\left(\mathrm{p}_{\mathrm{ti}} \mathrm{O}_{2}\right.$ and CMD). INM was begun, on average, $2.0 \pm 2.5$ days after hemorrhage. The treatment algorithm for DCI remained unchanged from 2010 to 2018. In the pre-INM group, 51 patients (53.1\%) were treated with iHTN versus $56(59.6 \%)$ in the
post-INM group $(\mathrm{p}=0.370)$. Endovascular rescue treatment was applied in 23 patients in the pre-INM group $(24.0 \%)$ versus 29 patients (30.9\%) in the post-INM group $(\mathrm{p}=0.266)$.

\section{Complications}

A nonsignificant trend toward a higher rate of CSF infections was observed in the post-INM group (20.2\% vs $11.5 \% ; \mathrm{p}=0.098$ ). No probes had to be removed due to signs of infection at the site of entry. A total of 10 hemorrhages were observed after probe implantation. Nine of the hemorrhages consisted of small bleeds along the trajectory of the probe. Operative evacuation was required in a single case. All of the hemorrhagic complications occurred under dual antiplatelet therapy after complicated or stent-assisted endovascular coiling. None resulted in permanent neurological deficits.

\section{Primary Outcome Parameter}

Twelve months after hemorrhage, a higher rate of favorable outcome was observed in the post-INM group compared to that of the pre-INM group (53.8\% vs 39.8\%), with more patients in upper moderate disability $(15.4 \%$ vs $6.8 \%)$ and lower good recovery (14.3\% vs $8.0 \%$ ) categories (Table 2 and Fig. 3). The binary logistic regression model of dichotomized data showed a trend toward better outcome for patients in the post-INM group (OR 0.60, $95 \%$ CI $0.33-1.09, \mathrm{p}=0.094)$. Ordinal logistic regression analysis (with modified Fisher grade, H\&H score, and DCI occurrence as predictor covariates) demonstrated a significant difference in the GOSE score distribution (OR $4.86,95 \% \mathrm{CI}-1.17$ to $-0.07, \mathrm{p}=0.028)$.

\section{Secondary Outcome Parameters}

At discharge, the GOSE score distribution showed that a favorable outcome was achieved in $38 \%$ of patients in the post-INM group compared to $25 \%$ in the pre-INM group (OR $0.08,95 \% \mathrm{CI}-0.44$ to $0.59, \mathrm{p}=0.776$ ). The GOSE distribution after 6 months is available in Supplementary Table 2 . The overall mortality rate, as well as the overall incidence of DCI, remained unchanged after INM implementation. However, in the post-INM group, the first treatment-triggering DCI event was detected on average 2.2 days earlier compared to any event in the pre-INM group $(\mathrm{p}=0.002)$. The rate of silent infarction decreased significantly from $33.3 \%$ to $12.8 \%(\mathrm{p}=0.001)$ after introduction of INM, as did the overall rate of DCI-related infarction (from $44.8 \%$ to $22.3 \%$; $\mathrm{p}=0.001$ ). The DCIcaused mortality rate did not differ significantly between the groups $(10.6 \%$ vs $14.6 \% ; \mathrm{p}=0.265)$. As a secondary effect, the need for CT imaging during clinical decisionmaking was reduced from on average 8.4 to 6.8 images per patient $(\mathrm{p}=0.038)$, resulting in a reduction in radiation exposure in the post-INM group.

\section{Discussion}

In SAH patients, the occurrence of DCI and DCIrelated infarction is directly correlated with clinical outcome. ${ }^{21}$ The current definition of delayed cerebral ische- 
TABLE 1. Baseline characteristics in 190 patients with poor-grade SAH

\begin{tabular}{|c|c|c|c|}
\hline Characteristic & Pre-INM $(n=96)$ & Post-INM $(n=94)$ & p Value \\
\hline Mean age in yrs (range) & $53.9 \pm 12.2(19-86)$ & $56.5 \pm 12.2(32-87)$ & 0.146 \\
\hline Female/male ratio & $73: 23$ & $70: 24$ & 0.802 \\
\hline \multicolumn{4}{|l|}{ Risk factor } \\
\hline Hypertension & $39(40.6 \%)$ & $41(43.6 \%)$ & 0.676 \\
\hline Smoking & $27(28.1 \%)$ & $30(31.9 \%)$ & 0.569 \\
\hline $\mathrm{BMl}$ in $\mathrm{kg} / \mathrm{m}^{2}( \pm \mathrm{SD})$ & $26 \pm 4.5$ & $26 \pm 5.0$ & 0.499 \\
\hline Aneurysm diameter max in mm ( \pm SD) & $7.6 \pm 3.8$ & $7.2 \pm 4.7$ & 0.564 \\
\hline $\mathrm{H} \& \mathrm{H}$ & & & 0.068 \\
\hline Grade 3 & $44(45.8 \%)$ & $58(61.7 \%)$ & \\
\hline Grade 4 & $34(35.4 \%)$ & $20(21.3 \%)$ & \\
\hline Grade 5 & $18(18.8 \%)$ & $16(17 \%)$ & \\
\hline Aneurysm location & & & 0.183 \\
\hline MCA & $36(37.5 \%)$ & $31(33.0 \%)$ & \\
\hline AComA & $24(25.0 \%)$ & $26(27.7 \%)$ & \\
\hline ICA & $15(15.6 \%)$ & $11(11.7 \%)$ & \\
\hline PComA \& AChA & $9(9.4 \%)$ & $9(9.6 \%)$ & \\
\hline BA & $5(5.2 \%)$ & $3(3.2 \%)$ & \\
\hline Others & $7(7.3 \%)$ & $14(14.9 \%)$ & \\
\hline Anterior circulation & $77(80.2 \%)$ & $73(77.7 \%)$ & \\
\hline Posterior circulation & $19(19.8 \%)$ & $21(22.3 \%)$ & \\
\hline Clipping/coiling & $49 / 47$ & $45 / 48$ & \\
\hline Modified Fisher scale & & & 0.068 \\
\hline Grade 1 & $8(8.3 \%)$ & $11(11.7 \%)$ & \\
\hline Grade 2 & $9(9.4 \%)$ & $11(11.7 \%)$ & \\
\hline Grade 3 & $43(44.8 \%)$ & $20(21.3 \%)$ & \\
\hline Grade 4 & $35(36.5 \%)$ & $52(55.3 \%)$ & \\
\hline \multicolumn{4}{|l|}{$\mathrm{DCl}$ surveillance } \\
\hline Neurologically assessable (days 4-14) & $45(46.9 \%)$ & $47(50.0 \%)$ & 0.667 \\
\hline INM & None & $55(58.8 \%)$ & \\
\hline $\mathrm{P}_{\mathrm{ti}} \mathrm{O}_{2}$ & None & $54(57.4 \%)$ & \\
\hline CMD & None & $44(46.8 \%)$ & \\
\hline
\end{tabular}

$\mathrm{ACh} A=$ anterior choroidal artery; $\mathrm{AComA}=$ anterior communicating artery; $\mathrm{BA}=$ basilar artery; $\mathrm{BMI}=$ body mass index; $\mathrm{ICA}=$ internal carotid artery; $\mathrm{MCA}=$ middle cerebral artery; $\mathrm{PComA}=$ posterior communicating artery.

Values are presented as the number (\%) of patients unless otherwise specified.

mia is solely based on clinical characteristics, precluding its use in poor-grade SAH patients. Additional concepts to evaluate the unconscious patient are therefore required. In a previous retrospective cohort analysis, we showed that INM is a powerful monitoring tool during endovascular DCI treatment. ${ }^{16}$ In this current two-group cohort analysis, the implementation of INM and the accompanying broadening of the classic DCI definition were associated with earlier detection of treatable DCI events. This resulted in earlier initiation of DCI treatment. Earlier treatment in the adequately selected patient resulted in a reduction of infarctions. This, in turn, translated into a higher rate of long-term favorable outcome in the post-INM group. At discharge, this difference is not yet apparent. However, a higher rate of upper and lower severe disability in the post-INM group was observed. These are patients with the rehabilitation potential of reaching a favorable out- come (e.g., lower and upper moderate disability) after 12 months. Analysis of the dichotomized data confirmed this trend, and the ordinal regression model of GOSE score distribution after 12 months demonstrated significant differences between the groups. In a subgroup analysis, it became clear that patients with $\mathrm{H} \& \mathrm{H}$ grade 3 hemorrhages benefited the most from INM application because they are at high risk of DCI development and have the most to lose.

As fewer CT scans were obtained in patients who underwent invasive monitoring, we believe that the technique can help to safely reduce the rate of imaging and thus exposure to ionizing radiation and potentially hazardous patient transports. ${ }^{22}$

Because treatment approaches to DCI vary among centers, our results cannot be readily extrapolated. Apart from prophylactic oral nimodipine, not a single treatment has been proven to be beneficial in a randomized trial on 
TABLE 2. Overview of results

\begin{tabular}{|c|c|c|c|}
\hline Outcome & Pre-INM (n = 96) & Post-INM ( $n=94)$ & $p$ Value \\
\hline \multicolumn{4}{|l|}{$\mathrm{DCl}$ diagnostics } \\
\hline $\mathrm{DCl}$ incidence & $55(57.3 \%)$ & $60(63.8 \%)$ & 0.357 \\
\hline $\begin{array}{l}\text { Median lag 1st } \mathrm{DCl} \text { event in days } \\
\quad( \pm \mathrm{SD})\end{array}$ & $7.5 \pm 5.3$ & $5.3 \pm 2.9$ & 0.002 \\
\hline Silent infarction & $32(33.3 \%)$ & $12(12.8 \%)$ & 0.001 \\
\hline DCl-related infarction & $43(44.8 \%)$ & $21(22.3 \%)$ & 0.001 \\
\hline Overall mortality & $20(20.8 \%)$ & $19(20.2 \%)$ & 0.916 \\
\hline DCl-related deaths & $14(14.6 \%)$ & $10(10.6 \%)$ & 0.265 \\
\hline \multicolumn{4}{|l|}{$\mathrm{DCl}$ treatment } \\
\hline iHTN & $51(53.1 \%)$ & $56(59.6 \%)$ & 0.370 \\
\hline ERT & $23(24.0 \%)$ & $29(30.9 \%)$ & 0.266 \\
\hline Angioplasty & $15(15.6 \%)$ & $10(10.6 \%)$ & \\
\hline Spasmolysis & $32(33.3 \%)$ & $34(36.2 \%)$ & \\
\hline Patients w/ multiple ERTs & $14(14.6 \%)$ & $15(16.0 \%)$ & \\
\hline \multirow[t]{2}{*}{ Total no. of ERTs } & 39 & 45 & \\
\hline & Pre-INM $(n=96)$ & Post-INM $(n=94)$ & \\
\hline Discharge GOSE result & & & 0.776 \\
\hline Death & $21(21.9 \%)$ & $19(20.2 \%)$ & \\
\hline Vegetative state & $9(9.4 \%)$ & $11(11.7 \%)$ & \\
\hline Lower severe disability & $31(31.3 \%)$ & $36(38.3 \%)$ & \\
\hline Upper severe disability & $19(19.8 \%)$ & $10(10.6 \%)$ & \\
\hline Lower moderate disability & $12(12.5 \%)$ & $10(10.6 \%)$ & \\
\hline Upper moderate disability & $4(4.2 \%)$ & $6(6.4 \%)$ & \\
\hline Lower good recovery & $0(0.0 \%)$ & $2(2.1 \%)$ & \\
\hline Upper good recovery & $0(0.0 \%)$ & $0(0.0 \%)$ & \\
\hline Median score (IQR) & $3(2-4)$ & $3(2-4)$ & \\
\hline \multirow[t]{2}{*}{ Favorable outcome, scores 5-8 } & $16(25 \%)$ & $18(38 \%)$ & 0.559 \\
\hline & Pre-INM $(n=88)$ & Post-INM $(n=91)$ & \\
\hline 12-mo GOSE result & & & 0.028 \\
\hline Death & $25(28.4 \%)$ & $22(24.2 \%)$ & \\
\hline Vegetative state & $7(8.0 \%)$ & $5(5.5 \%)$ & \\
\hline Lower severe disability & $8(9.1 \%)$ & $6(6.6 \%)$ & \\
\hline Upper severe disability & $13(14.8 \%)$ & $9(9.9 \%)$ & \\
\hline Lower moderate disability & $17(19.3 \%)$ & $15(16.5 \%)$ & \\
\hline Upper moderate disability & $6(6.8 \%)$ & $14(15.4 \%)$ & \\
\hline Lower good recovery & $7(8.0 \%)$ & $13(14.3 \%)$ & \\
\hline Upper good recovery & $5(5.7 \%)$ & $7(7.7 \%)$ & \\
\hline Median score (IQR) & $4(1-5)$ & $5(2-6)$ & \\
\hline Favorable outcome, scores $5-8$ & $35(39.8 \%)$ & $49(53.8 \%)$ & 0.094 \\
\hline
\end{tabular}

$\mathrm{ERT}=$ endovascular rescue therapy.

Values are presented as the number (\%) of patients unless otherwise specified. Boldface type indicates statistical significance. The GOSE outcome categories are defined as follows: $1=$ death; 2 = vegetative state (unable to obey commands); 3 = lower severe disability (dependent on others for care); $4=$ upper severe disability (independent at home); 5 = lower moderate disability (independent at home and outside the home but with some physical or mental disability); $6=$ upper moderate disability (independent at home and outside the home but with some physical or mental disability, with less disruption than lower moderate disability); $7=$ lower good recovery (able to resume normal activities with some injury-related problems); 8 = upper good recovery (no problems). 


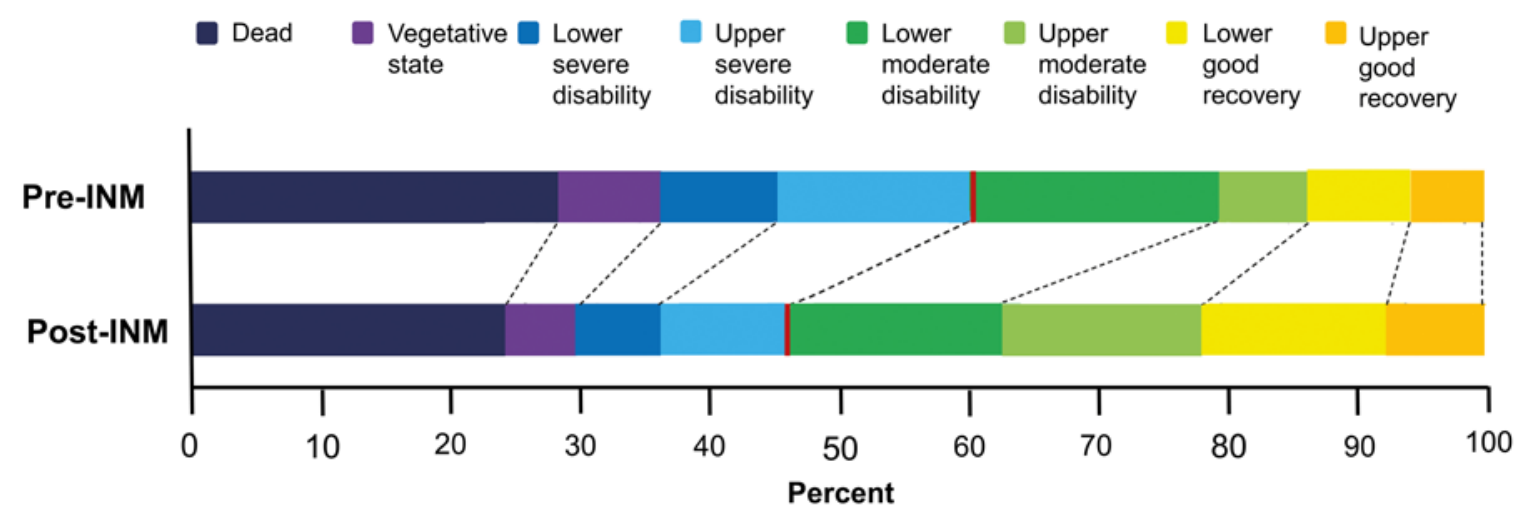

FIG. 3. Stacked bar chart of GOSE results after 12 months in the pre-INM and post-INM groups.

outcome in SAH patients. The HIMALAIA study has questioned the use of induced hypertension but was terminated due to a lack of recruitment. The application of euvolemic hypertension remains largely based on observational data. ${ }^{23}$ With this in mind, there is still no class I evidence to support our current practice. The evidence for endovascular rescue treatment is even more scarce and mostly based on case series. We have shown in the past that endovascular rescue therapy can be safe and effective in highly selected patients. ${ }^{17}$ The evaluation of long-term clinical outcome will be the topic of future analyses. The improvement of diagnostic abilities is only as valuable as the efficacy of the treatment approaches used. Nevertheless, with our treatment algorithm itself remaining unchanged between groups, the improvement in the detection rate of DCI and in outcome can be appreciated and attributed to the change in monitoring.

Treatment guidance with invasive monitoring has three main potential pitfalls: the limited spatial resolution of local information, the problem of a cutoff value, and technical difficulties. Implanted probes only provide information from brain tissue in their immediate proximity. We have observed patients developing distinct contralateral or posterior fossa ischemia without registration of aberrant measurements. Whether a $\mathrm{p}_{\mathrm{ti}} \mathrm{O}_{2}$ sensor or microdialysis probe in the anterior watershed region is able to detect impeding ischemia in the posterior portion of the middle cerebral artery territory is unclear. However, a thresholdbased approach as such is an oversimplification, not taking into account the dynamic and more complex nature of the underlying pathophysiological mechanisms. The relative changes toward baseline values and the slopes of parameter curves may be of greater importance and will be part of a future analysis. Technical difficulties with $\mathrm{p}_{\mathrm{ti}} \mathrm{O}_{2}$ sensors, and especially microdialysis probes, are not uncommon. Erroneous measurements can lead to prejudicial treatment decisions, and unreliable probes have to be identified early.

Complications of probe implantation are seldom serious. We observed 9 microbleeds that required no further treatment and 1 hemorrhage necessitating surgical evacuation. No neurological sequelae were observed. All patients with hemorrhagic complications were under double antiplatelet treatment, emphasizing the need for placement before endovascular aneurysm closure. The higher rate of
CSF infections in the post-INM group needs to be critically addressed. These were patients with a positive CSF culture and an increased cell count collected from an external ventricular drain. It is difficult to assess the contribution of adding an additional port of contamination by INM as all patients also had an external ventricular drain. It is worth noting, however, that no probe had to be removed due to local signs of infection at the site of entry.

This trial is part of a broader effort to extend the classic definition of DCI by including functional aspects of perfusion, oxygenation, and metabolism.

\section{Limitations}

Despite having one of the largest and most comprehensively recorded cohorts of SAH patients, this study is limited by an observational design where both study groups are separated in time. Individuals were included according to the predefined inclusion and exclusion criteria. However, outcome data of the pre-INM group had to be collected retrospectively. This introduces an information bias that cannot be corrected. Reports of clinical SAH grading and the documentation of INM application have been very rigorous in minimizing any selection bias. However, there are still multiple potential confounders and sources of effect modification that cannot be corrected. Also, simply inclusion into the study of post-INM patients creates a context of general higher vigilance, potentially contributing to earlier DCI detection independent of invasively collected data and therefore creating detection bias. Finally, outcome data assessment could not be blinded, creating potential observer and information bias. Comparability between the groups was, however, tested extensively and was confirmed without significant differences, negating the need for additional matching.

\section{Conclusions}

In this study, INM with a resulting extension of the classic DCI definition toward a functional dimension enabled earlier detection of treatable DCI events in poor-grade SAH patients. This translated into a reduction of silent and overall DCI-related infarctions and an improvement in outcome after 12 months. 


\section{References}

1. Dodel R, Winter Y, Ringel F, et al. Cost of illness in subarachnoid hemorrhage: a German longitudinal study. Stroke. 2010;41(12):2918-2923.

2. Vergouwen MD, Etminan N, Ilodigwe D, Macdonald RL. Lower incidence of cerebral infarction correlates with improved functional outcome after aneurysmal subarachnoid hemorrhage. J Cereb Blood Flow Metab. 2011;31(7):15451553.

3. Macdonald RL. Origins of the concept of vasospasm. Stroke. 2016;47(1):e11-e15.

4. Macdonald RL, Higashida RT, Keller E, et al. Clazosentan, an endothelin receptor antagonist, in patients with aneurysmal subarachnoid haemorrhage undergoing surgical clipping: a randomised, double-blind, placebo-controlled phase 3 trial (CONSCIOUS-2). Lancet Neurol. 2011;10(7):618-625.

5. Pickard JD, Murray GD, Illingworth R, et al. Effect of oral nimodipine on cerebral infarction and outcome after subarachnoid haemorrhage: British aneurysm nimodipine trial. BMJ. 1989;298(6674):636-642.

6. Rabinstein AA, Friedman JA, Weigand SD, et al. Predictors of cerebral infarction in aneurysmal subarachnoid hemorrhage. Stroke. 2004;35(8):1862-1866.

7. Weidauer $\mathrm{S}$, Lanfermann $\mathrm{H}$, Raabe A, et al. Impairment of cerebral perfusion and infarct patterns attributable to vasospasm after aneurysmal subarachnoid hemorrhage: a prospective MRI and DSA study. Stroke. 2007;38(6):1831-1836.

8. Anzabi M, Angleys H, Aamand R, et al. Capillary flow disturbances after experimental subarachnoid hemorrhage: a contributor to delayed cerebral ischemia? Microcirculation. 2019;26(3):e12516.

9. Balbi M, Koide M, Wellman GC, Plesnila N. Inversion of neurovascular coupling after subarachnoid hemorrhage in vivo. J Cereb Blood Flow Metab. 2017;37(11):3625-3634.

10. Liu H, Dienel A, Schöller K, et al. Microvasospasms after experimental subarachnoid hemorrhage do not depend on endothelin A receptors. Stroke. 2018;49(3):693-699.

11. Terpolilli NA, Brem C, Bühler D, Plesnila N. Are we barking up the wrong vessels? Cerebral microcirculation after subarachnoid hemorrhage. Stroke. 2015;46(10):3014-3019.

12. Vergouwen MD, Vermeulen M, van Gijn J, et al. Definition of delayed cerebral ischemia after aneurysmal subarachnoid hemorrhage as an outcome event in clinical trials and observational studies: proposal of a multidisciplinary research group. Stroke. 2010;41(10):2391-2395.

13. Sarrafzadeh AS, Vajkoczy P, Bijlenga P, Schaller K. Monitoring in neurointensive care-the challenge to detect delayed cerebral ischemia in high-grade aneurysmal SAH. Front Neurol. 2014;5:134.

14. Bricout N, Estrade L, Boustia F, et al. Reduced-dose CT protocol for the assessment of cerebral vasospasm. Neuroradiology. 2015;57(12):1211-1218.

15. Hutchinson P, O'Phelan K. International multidisciplinary consensus conference on multimodality monitoring: cerebral metabolism. Neurocrit Care. 2014;21(suppl 2):S148-S158.

16. Albanna W, Weiss M, Müller M, et al. Endovascular rescue therapies for refractory vasospasm after subarachnoid hemorrhage: a prospective evaluation study using multimodal, continuous event neuromonitoring. Neurosurgery. 2017;80(6):942-949.

17. Weiss M, Conzen C, Mueller M, et al. Endovascular rescue treatment for delayed cerebral ischemia after subarachnoid hemorrhage is safe and effective. Front Neurol. 2019;10:136.

18. Jennett B, Snoek J, Bond MR, Brooks N. Disability after severe head injury: observations on the use of the Glasgow Outcome Scale. J Neurol Neurosurg Psychiatry. 1981;44(4):285293.
19. Wilson JT, Pettigrew LE, Teasdale GM. Structured interviews for the Glasgow Outcome Scale and the extended Glasgow Outcome Scale: guidelines for their use. J Neurotrauma. 1998;15(8):573-585.

20. Ilodigwe D, Murray GD, Kassell NF, et al. Sliding dichotomy compared with fixed dichotomization of ordinal outcome scales in subarachnoid hemorrhage trials. J Neurosurg. 2013;118(1):3-12.

21. Vergouwen MD, Ilodigwe D, Macdonald RL. Cerebral infarction after subarachnoid hemorrhage contributes to poor outcome by vasospasm-dependent and -independent effects. Stroke. 2011;42(4):924-929.

22. Bergman LM, Pettersson ME, Chaboyer WP, et al. Safety hazards during intrahospital transport: a prospective observational study. Crit Care Med. 2017;45(10):e1043-e1049.

23. Gathier CS, van den Bergh WM, Slooter AJ. HIMALAIA (Hypertension Induction in the Management of AneurysmaL subArachnoid haemorrhage with secondary IschaemiA): a randomized single-blind controlled trial of induced hypertension vs. no induced hypertension in the treatment of delayed cerebral ischemia after subarachnoid hemorrhage. Int $J$ Stroke. 2014;9(3):375-380.

\section{Disclosures}

Dr. Wiesmann is a consultant for Stryker Neurovascular. He receives honoraria from Braco Imaging, Medtronic, Penumbra, Siemens Healthcare, and Stryker Neurovascular. He receives support of non-study-related clinical or research efforts that he oversees from the following: Abbott, ab medica, Acandis, Asahi Intecc, Bayer, Bracco Imaging, B. Braun, Cerenovus, Codman Neurovascular, Dahlhausen, Kaneka Pharmaceuticals, Medtronic, Mentice AB, MicroVention, Penumbra, Phenox, Philips Healthcare, Route 92, Siemens Healthcare, SilkRoad Medical, St. Jude, and Stryker Neurovascular.

\section{Author Contributions}

Conception and design: Veldeman, Albanna, Schubert. Acquisition of data: all authors. Analysis and interpretation of data: Veldeman, Schubert. Drafting the article: Veldeman, Albanna. Critically revising the article: all authors. Reviewed submitted version of manuscript: Veldeman, Albanna, Weiss, Conzen, Schmidt, Wiesmann, Clusmann, Schubert. Approved the final version of the manuscript on behalf of all authors: Veldeman. Statistical analysis: Veldeman. Administrative/ technical/material support: Veldeman, Albanna, Weiss, Conzen, Schmidt, Schulze-Steinen, Clusmann, Schubert. Study supervision: Clusmann, Schubert.

\section{Supplemental Information}

\section{Online-Only Content}

Supplemental material is available with the online version of the article.

Supplementary Tables 1 and 2. https://thejns.org/doi/suppl/ 10.3171/2020.3.JNS20375.

\section{Correspondence}

Michael Veldeman: RWTH Aachen University, Aachen, Germany. mveldeman@ukaachen.de. 